\title{
Superstring Symmetry Breaking Through Induced String Vibrations
}

\author{
AC Tahan ${ }^{1}$ \\ ${ }^{1}$ ETHERMED $_{\mathrm{TM}}$, Cambridge, MA, USA \\ Correspondence: AC Tahan, ETHERMED, PO Box 391987, Cambridge, MA 02139, USA. E-mail: \\ actahan@ethermed.com
}

Received: March 2, 2015

Accepted: March 20, $2015 \quad$ Online Published: April 11, 2015

doi:10.5539/esr.v4n2p35

URL: http://dx.doi.org/10.5539/esr.v4n2p35

\begin{abstract}
Strings have been recorded in the laboratory (Tahan 2011). The responsible string experimentation method involves a symmetry breaking that permits access to a fifth dimension of gravitons and superpartners (Tahan 2012, 2013, 2014). The symmetry breaking can be thought opposite to traditional understanding since it introduces superparticles to the Standard Model visible sector.
\end{abstract}

Keywords: superstring symmetry breaking, fifth dimension, graviton, supersymmetry, brane, string, black hole

\section{Introduction}

Recent detection of a particle with the characteristics of the Standard Model Higgs Boson (CMS Collaboration 2014, 2013, 2012, ATLAS Collaboration 2012) alluded to the importance of symmetry breaking for the observable Universe; individual characteristics for the weak force and electromagnetism bosons would not exist otherwise. The discovery allows the Standard Model to well-explain the particles and forces of Nature, except for gravity. Proposals for attributing a boson to gravity while explaining the hierarchy problem have included string theory and extra dimensions.

D-branes with gauge bosons originating from open strings with ends restricted to the branes and closed strings as gravitons living in an extra-dimensional bulk (Meissner, Nilles, \& Olechowski 1999) are well-discussed in the literature. However, no observation or experimental datum for strings was preventing acceptance of string theory as an underlying explanation for the Universe.

An innovation is allowing for research with strings (Tahan 2011), permitting exploration of the Standard Model in relation to extra dimensions. Experimentation with the device suggested that the proton is a brane structure with tension that can be affected with a magnetic field. The manipulation of the proton brane was the basis for a symmetry breaking that permitted access to gravitons and superparticles that influenced spacetime, resulting in the recording of a D-brane with an open string for visible light (Tahan 2011). The events in the lab related to graviton emergence including black holes and the D-branes were suggestive of extra dimensions.

This manuscript discusses in more detail than what has been published on string experimentation the symmetry breaking ability of the string experimentation method, which extends consideration of symmetry breaking to involve strings. The string experimentation symmetry breaking--superstring symmetry breaking--causes particles to appear that have been excluded from the Standard Model due to a symmetry breaking. Superstring symmetry breaking is a process permitting observation of gravity bosons and supersymmetry somewhat analogous to electroweak symmetry breaking allowing appearance of the weak force and electromagnetism. The method involves the Higgs mechanism in the breaking of the structure or symmetry of D-branes to access an extra dimension from which gravitons and superparticles that are unobserved can emerge. The symmetry breaking is confirmation of supersymmetric gauge theory through D-branes at low energy.

The string experimentation method briefly will be explained before describing the resulting area of superstring symmetry breaking, including related to the creation of string black holes. This work is based on experiments, and though the manuscript is not a typical theoretical or math paper focused on equations it involves topics that have been examined traditionally mathematically--e.g. symmetry, QCD, mass gap. The experimentation in the context of the math work may progress studies; the math community should understand that strings are no longer purely a mathematical consideration. The paper presents a laboratory technique for symmetry breaking involving string vibrations, which theorists could understand as a demonstration of how superstring symmetry can be broken without the need for perturbation theory. 


\section{Revisiting String Experimentation}

Associating mass with gauge fields (Schwinger 1962) led to the idea of discussing mass in the context of the strong force field (Anderson 1963), which can be thought had been a natural progression from consideration of non-Abelian gauge theory in the context of SU(2) symmetry (Yang \& Mills 1954). Accordingly, the Higgs mechanism that provides gauge bosons with mass (Higgs, Phys. Lett. 1964, Phys. Rev. Lett. 1964, 1966, Englert \& Brout 1964, Guralnik, Hagen, \& Kibble 1964) surfaced shortly afterward. String experimentation means the use or observation of strings in experiments and has been a study of mass in QCD by considering strings to underlie the theory, a topology of gluon strings with quarks at the ends ('t Hooft 1974) that permits the unbroken gauge symmetry (Weinberg 1973, Gross \& Wilczek 1973) to become broken through photon absorption. Prior to work with the Figure 1 set-up, scientists believed visualization of strings in the laboratory would be impossible due to required high energy. String experimentation sources energy from the proton brane to create symmetry breaking. The concept requires acceptance that energy is synonymous to a string and growing string vibrations are equivalent to an increasing energy (Scherk \& Schwarz, Nucl. Phys. B 1974).

\subsection{Set-Up}
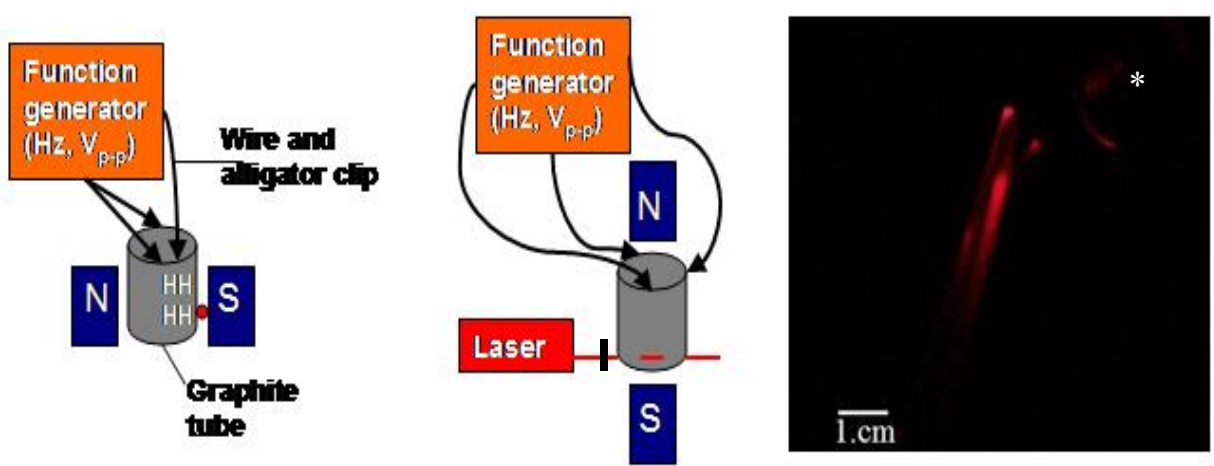

Figure 1. String experimentation set-up and appearance of a D-brane with an open string

The three images are the experimental set-up and the largest D-brane with an open string from trials. The first drawing presents the set-up as though viewed from the front. $\mathrm{N}$ and $\mathrm{S}$ are the poles of a gap magnet. The red dot is the approximate position of the laser on the side of the tube when the laser pointer (Quartet Standard Laser Pointer) was incorporated with the set-up. The laser was not part of the string experimentation method and was only used to observe if productions from the set-up would influence the path of the light toward the graphite tube. Removing the red dot from the first picture would present the complete string experimentation set-up with Hydrogen in the tube. The middle image can be thought more of an above the set-up presentation similar to the perspective of the camera that was clamped above the set-up and recorded the largest D-brane with an open string, though the complete tube is illustrated. The black line on the red line that represents the path of the laser to the tube provides an idea of the location where D-branes appeared, approximately $.01-.02 \mathrm{~m}$ from the graphite tube. In other words, the D-brane with an open string for the laser light in the middle of the third picture, a frame from a video, can be considered to have been recorded at the black line position due to influences from the tube affecting spacetime (Tahan 2011, 2015). The curved light near the upper right corner of the third picture is the laser light bending around the graphite tube, the light having been initially a red line or dot (Tahan 2011). The light to the immediate left of the asterisk in the third picture is the suspected location of a black hole that occurred in the trial, the light being from the laser and having entered the tube due to the high gravitational area (Tahan 2011).

The experiments were studies of Hydrogen. Sulfuric acid $\left(\mathrm{H}_{2} \mathrm{SO}_{4}\right)$ (typically $20 \mathrm{~mL}, 96 \%$ concd. Mallinckrodt Analytical Reagent, ACS) as the proton source was placed in a graphite tube (Crucible, Saed/Manfredi G40, 1.5 "OD x 1.25 "ID x 3.75 "DP) for each experiment involving the laser. Approximately $99.7 \mathrm{kPa}$ and $98-100 \%$ were the barometric pressure and relative humidity (Boston, MA, CW1378) during the largest laser light D-brane trial (Tahan 2011). The tube was clamped in an approximately 2000Gs, static magnetic field consistently near the $\mathrm{S}$ pole of the gap magnet. Small distance variations toward the $\mathrm{S}$ pole were immaterial, having the tube at a location measuring $2000 \mathrm{Gs}$ being the only objective. About $2 \mathrm{~Hz}(2.000-2.012 \mathrm{~Hz})$ and $\mathrm{V}_{\mathrm{p}-\mathrm{p}}=4.312-4.437$, predominately 4.375 , were supplied to the graphite container through wires from a breadboard to which a function generator was attached by red and black alligator clips to a LED. The low frequency was not intended to alter Hydrogen alignment in the magnetic field. Though only one wire from the breadboard was needed, three 
wires with alligator clips were connected triangularly to the top of the tube to be sure that the frequency and amplitude were being supplied--considering potential inoperability in a trial due to alligator clip corrosion from acid exposure.

The components always needed are the frequency and amplitude, tube, constant magnetic field strength $(\approx 2000 \mathrm{Gs})$, and concentrated sulfuric acid. Diverse manners for supplying the parts can be tested, e.g. a different Hydrogen source, but modifications to the components may be necessary depending on the change: for example the amplitude may need adjustment. Again the laser was only incorporated in trials to visualize the altering of the light path, as by gravitons interacting with the graphite tube (Tahan 2011). It did not have a role in the symmetry breaking process. The graphite tube and acid supply should be changed occasionally to avoid variables, for instance impurities in the acid or eventual deformation of the tube due to the acid that may inhibit regular frequency conduction. The acid should be replaced in the tube for each trial unless the examiner wishes to test a particular aspect of the set-up involving the acid.

\subsection{Background}

Initial experiments to produce isotopes involving Hydrogen or Hydrogen with inserted powdered elements in the Hydrogen source $\mathrm{H}_{2} \mathrm{SO}_{4}$ suggested that Hydrogen is a rectangular membrane structure with tension that can be altered in magnetic fields, which led to a consideration of strings. By that, the membrane or brane idea emerged from exposing the element to different magnetic fields and frequencies and finding that specific extremely low radio wave frequencies concluded to be resonant frequencies were allowing for isotope productions. Pushing and pulling influences had been observed in preliminary trials involving a loosely hanging Pasteur pipette near the holding vessel for the sulfuric acid, which led to the isotope and laser light work. The thought became that low frequencies with particular amplitudes were not only involved with the release of Hydrogen from the proton donating acid but the related photons were affecting individual Hydrogen since the influences were seen at specific times in the roughly one hour trials: a pushing influence typically preceding a pulling affect.

Various control trials to understand what might be causing the events were performed. A Germanium detector was incorporated with the set-up, which apart from presenting readings for isotope productions led to the conclusion that the holding vessel was releasing energy at the same times as the pushing and pulling observations. Thus, the laser light was incorporated with the set-up (Tahan 2011) to understand if the path of the light would change due to influences from the tube. In other words, the pushing and pulling influences were puzzling since control trials did not provide explanations or alternatives to the extreme thought that gravitons possibly were being released due to the set-up and interacting with the tube, thereby bending spacetime and consequently moving the less massive pipette. The energy at the times of the pushing and pulling observations were thought potentially related to the process of graviton emergence.

The isotope work that suggested the proton to be a brane structure allowed for the thought of gravitons since string theory is a theory for quantum gravity (Scherk \& Schwarz, Phys. Lett. 1974). The initial hypothesis for the laser light work was that a frequency with a particular amplitude could create vibrations of the Hydrogen brane that would be equivalent to an increasing energy to a level that had resulted in the pushing and pulling observations. The same frequency for the isotope and pipette work with the approximately 2000Gs magnet would be used. Change in the path of the light would suggest that the pipette movements had been due to particle release from Hydrogen with the nature of gravity: gravitons. The idea was that altering of the travel of the light would be caused by bending of spacetime resulting from added mass-energy to the graphite tube from interacting gravitons that had materialized from Hydrogen.

The bending of the laser light around the tube, including the visualization of a D-brane with an open string for the laser light (Tahan 2011), supported that gravitons were being released and that an extra dimension was being accessed, likely the fifth dimension or bulk that is often discussed in literature. Preliminary trials, e.g. involving polarizers, suggested that the pushing influence had photon characteristics that supported that the affect was due to the superparticle graviphoton. Accordingly, also gravitinos likely emerged (Tahan 2013). Therefore, the conclusion became that low frequency with specific amplitude created vibrations toward a specific limit, where symmetry breaking occurred thereby providing access to the fifth dimension from which gravitons and superparticles surfaced to the Standard Model visible sector. The energy for the symmetry breaking was due to vibrations in the proton; the extra dimension was being accessed through use of the proton brane.

The main conclusion from experiments continued to be that the foundation of Hydrogen is the string, principally gluon field strings, with tension that can be altered with a magnetic field thereby permitting increased vibrations due to electromagnetic energy of the proper frequency in consideration of the manipulated tension. $2 \mathrm{~Hz}$ $(2.000-2.012 \mathrm{~Hz})$ and $\mathrm{V}_{\mathrm{p}-\mathrm{p}}=4.312-4.437$, predominately 4.375 , with the graphite container in a 2000Gs gap 
magnet was used for the majority of trials; the graphite tube was used for all laser light work. Hydrogen maintaining alignment in the magnetic field caused the tension of the Hydrogen brane and associated strings to stay continuous thus allowing for growing vibrations.

\subsection{Superstring Symmetry Breaking}

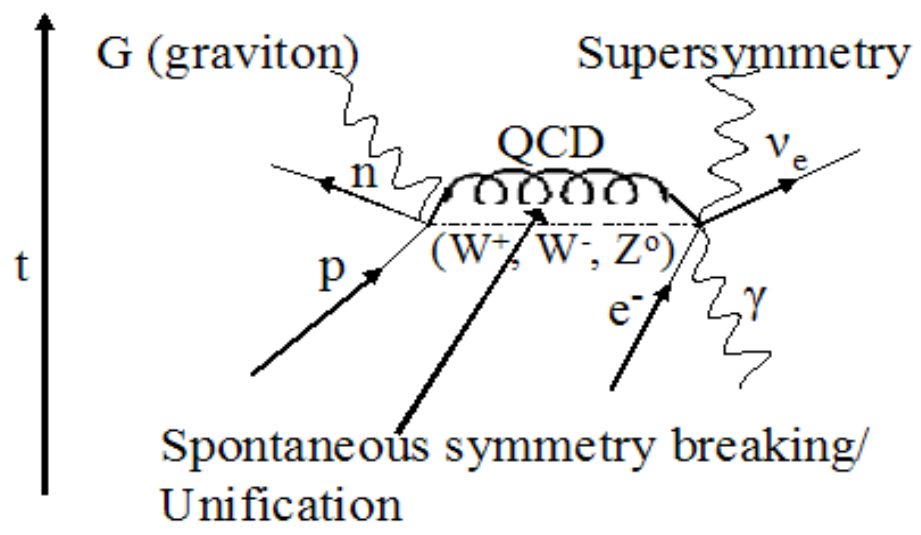

Figure 2. Gravitons and supersymmetry due to string experimentation

The diagram illustrates the string experimentation process that permits periodic appearances of superpartners and gravitons in the laboratory and can be presented as a reaction: gluon, gluon, gluon, gluino, gluino, gluino, photon, proton, electron, $\mathrm{W}^{-}$boson, Higgs boson, $\mathrm{Z}$ boson, axion, dilaton $\rightarrow$ graviton, gravitino, neutron, electron neutrino, graviscalar, graviphoton, photon, photon, photon, sneutrino, selectron, saxion (Tahan 2012). Incorporating particular detectors with the Figure 1 set-up should lead to refinement of the reaction that regards chief bodies associated with the symmetry breaking involving the Higgs boson and should not be believed a complete representation of all particles that can emerge due to the string experimentation method, which can be explored with the detectors.

Figure 2 diagrams the symmetry breaking method. The positions of the symmetry breakings in the tubes of the experimental set-up were understood when high gravitational areas--black holes--appeared, which could be great enough to cause redshifting of the laser light and frame dragging (Tahan 2011). The presentation shows where the symmetry breaking occurred to be a location of unification of the forces of Nature; the four forces are indistinguishable. The location is where deconfinement of the proton, glueball materialization, and consequently the Higgs boson emerged that resulted in access to the fifth dimension. The area can be understood as a merged collection of strings from the forces that was possible due to the string experimentation process with Hydrogen and symmetry breaking.

The symmetry breaking involving the Hydrogen brane was a consideration of Yang-Mills theory, a quantum gauge theory with the gauge group $\mathrm{SU}(\mathrm{N})$ being equivalent to a string theory with $1 / \mathrm{N}$ as the string coupling constant ('t Hooft 1974, Maldacena 1998). The 2Hz mainly influenced the gluon field string: a strong force string underlying a uniform field of quanta (Yang \& Mills 1954). Photons could be absorbed since the boson does not couple directly to the color gauge field SU(3) (Georgi \& Glashow 1974). The field strength (coupling constant) between quarks was consistent (Mills 1994); as energy increased the coupling constant decreased (Aharony, Gubser, Maldacena, Ooguri, \& Oz 2000). Considering N as a free parameter, the strong force coupling constant steadily reached a $\mathrm{N}$ analogous to a string vibration limit (Aharony, Gubser, Maldacena, Ooguri, \& Oz 2000) because of the incessant $2 \mathrm{~Hz}$ quanta resulting in deconfinement, possible due to the dual nature of SU(3): an unbroken non-Abelian gauge symmetry. Particles involved in the process are presented in the Figure 2 reaction (Tahan 2012), which should be edited in future studies by using specific detectors with the experimental set-up.

String experimentation is a method for emergence of the Higgs boson through QCD. Specifically, when understanding strings to be responsible for the make-up of Hydrogen the super-Higgs mechanism (Volkov \& Soroka 1973; Schwarz 1980) can be seen, a superstring symmetry breaking allowing for comprehension of the mass gap when appreciating that mass and confinement are different facets of gluon strings (Fradkin \& Shenker 1979). By that, confinement and the super-Higgs mechanism were concluded aspects of the strong force, a duality, when considering the glueball appearing due to the combining of the gluon strings (Tahan 2012). The symmetry breaking was a gateway to the extra dimension that contains superparticles and gravitons. The nearly 
parallel positioning of the largest laser light D-brane relative to the graphite tube (Tahan 2011) suggested that the fifth dimension is ubiquitous and all D-branes are in the bulk or fifth dimension (Tahan 2013). The brane structure of the proton as a separation to the fifth dimension became unstable since the gluon field strings as the main structural foundation of the brane no longer were in place.

The strong force can be understood short ranged if not described apart from strings. In keeping with Yang-Mills theory, every state of the system of strings has an energy of at least delta. Consequently, the vibration of strings or state of the system of string vibrations--movement or frequency--is massive relatively. Apart from remembering energy and string to be synonymous, the emergence of mass $(\mathrm{m})$ can be pictured by recalling frequency to have an association to energy: $\mathrm{E}=2 \pi \hbar f, \mathrm{E}=\mathrm{mc}^{2} \rightarrow \mathrm{m}=(2 \pi \hbar \mathrm{f}) / \mathrm{c}^{2}$. The well-known, simplistic relation creates visualization of the materialization of quanta from the string; quantum mechanics is emergent. Gluon strings are massive since the bodies are continuously moving. Or mass is a facet of the gluon string, which again was understood with string experimentation due to the appearance of the Higgs boson when gluons combined after a maximum in string vibrations was reached (Tahan 2014). Accordingly, string experimentation involving the Hydrogen brane leading to symmetry breaking and the fifth dimension was possible due to QCD low energy confinement.

Again superstring symmetry breaking is a gluon fusion process, which is a well-accepted means for Higgs boson production (Campanario \& Kubocz 2013; LHC Higgs Cross Section Working Group 2013). However, rather than forcing gluon coalescence through a collision that also results in the uncontrolled exiting of other particles from the collision area, the string experimentation method focuses on individual Hydrogen. Mass-energy was added to the brane structure through the $2 \mathrm{~Hz}$ quanta. The fundamental structure of the Hydrogen (proton) ceased to exist due to the gradually increasing mass. The mass scale for superstring symmetry breaking can be low (Krasnikov 1987); the supersymmetry breaking scale has been presented can be near 10GeV (Schwarz 1982). The emergence of the Higgs mechanism--more accurately super-Higgs mechanism--through QCD strings can be thought to have been a demonstration of the string to be a unified structure.

Events resulting from the string stimulation supported that the Standard Model occurs from superstring theory (Scherk \& Schwarz 1975, Zwiebach 2004): a low energy phenomenology from a higher energy theory with symmetry breaking separating the energy scales. The D-brane with the open string observation for the laser light showed that a 2-brane can be the background structure for environments in which gauge bosons propagate. Influences from graviphotons have been theorized would support a 2-brane to be a solution for quantum M-theory ( $\mathrm{D}=11$ ) (Billó et al. 2000, Duff \& Stelle 1991, Bergshoeff, Sezgin, \& Townsend 1987) that compactifies $(\mathrm{D}=10)$ (Huq \& Namazie 1985, Hořava \& Witten, 460B 1996) with eleven dimensional supergravity being the minimal energy limit (Hořava \& Witten, 475B 1996). The laser light D-brane encouraged the need to consider dimensional compactification, apart from the earlier work suggesting that the pushing affects in the laboratory involved graviphotons. Thus, compactification radius likely influenced the mass scale so that the string experimentation method could have caused string vibrations equivalent to the Planck mass $10^{19} \mathrm{GeV}$ (Schwarz 1982) for superstring symmetry breaking. Yet, consideration of a low mass gravitino (Tahan 2013, 2014, Morita, Nakano \& Shimomura 2012, Takayama \& Yamaguchi 2000) suggested a lower breaking scale (Martin 2011). The string experimentation method involved the proton wherein the mass scale was limited to $\sim 10^{15}-10^{16} \mathrm{GeV}$ (Beringer et al. 2012). If returning to consideration of $\mathrm{N}$ in terms of quantized dual strings for the color gauge group $\mathrm{SU}(\mathrm{N})$, the vibration limit being set by the proton mass scale allows for addressing the gluon deconfinement process as proton decay. If the string vibrations reached $\sim 10^{15}-10^{16} \mathrm{GeV}$, unification as labeled in Figure 2 should be thought a grand unification. The altered tension due to the magnetic field should be remembered to have been necessary for a specific vibration or mass scale to be achieved.

The proton can be pictured to be intact as the gluon strings combine, thereby suddenly eliminating the proton structure. The symmetry breaking or access to the fifth dimension was fleeting, concluded with lab observations of the transient influences from gravitons and superparticles. Emergence of the neutron, probably involving the electron in the string experimentation process (Tahan 2012), was confirmed in trials with isotope productions due to neutron capture.

\section{Black Holes}

The black holes in the graphite tube were not equivalent to the typical formation of a collapsed star but were the high mass-energy areas that appeared due to the string experimentation process with graviton emergence from the fifth dimension contributing to the locations. Entrance to the fifth dimension occurred because the underlying consistent symmetry for the proton was broken, i.e. complete separation between the Standard Model visible sector and the fifth dimension at specific Hydrogen no longer existed. Therefore, gravitons and superparticles 
could escape to the visible sector, which is how high gravitational areas or black holes happened in the tube. Wherever a black hole existed is where symmetry breaking involving a Hydrogen had occurred, which can be better visualized when remembering that all branes are immersed in the fifth dimension (Tahan 2013).

The black holes can be thought to have been a collection of strings, superparticles and gravitons but also the strings that made-up the Hydrogen thereby having been distinctive string regions (Lunin \& Mathur 2002). In other words, strings specific to the Hydrogen were also in the area so that the black holes were unique related to the initial structure under examination with the Figure 1 set-up. The black holes had a consistent string nature and can be described to have been areas of unification of the forces of Nature. The four forces were indistinguishable; the black holes were collective regions of strings that evaporated, e.g. string bodies as gravitons and superparticles having been released in trials (Tahan 2011). The black holes at the points of symmetry breaking in all trials were about the same sizes due to the invariable Figure 1 method unless the gravitons interacted with a larger number of atoms in the tube, which occurred in the trial with the largest laser light D-brane when the gravitons encountered excess graphite.

The fleeting fifth dimensional access can be imagined with an aspect of a different brane as the Universe brane covering thereby eliminating the opening just as water will fill a hole that suddenly appears in it. The gravitons and superparticles that surface at the time of the symmetry breaking, fifth dimensional access, will remain influential until the particles--i.e. energies of the bodies--completely dissipate to surroundings. The transient nature was understood by observing the influence the tube had on its environment, especially when considering the contribution of energy from the greatest high gravitational area--black hole--when the largest D-brane with an open string was observed (Tahan 2015).

Again larger black holes could be created by having gravitons interact with atoms in the tube, the biggest gravitational area in trials having been due to gravitons encountering graphite atoms. Additionally multiple symmetry breakings simultaneously could occur, especially by isolating more Hydrogen in the tube from the $\mathrm{H}_{2} \mathrm{SO}_{4}$ with DC current (Tahan 2011) thereby allowing for more superparticles and gravitons to appear concurrently. Trials involving directing superparticles and gravitons from the tube to objects as a pipette and water drops led to the conclusion that bodies radiated energies, e.g. gravity bosons were released or exchanged. Understanding that the same number of superparticles and gravitons emerged consistently per Hydrogen symmetry breaking, the extra graphite in the acid created more influences for visualizations because the graphite radiated superparticle and graviton energies. The gravitons increased string vibrations in the graphite atoms, which resulted in the atoms gaining mass-energy. Considering that other molecules in the acid were typically imparted with superparticle and graviton energies a collective area of high gravitation emerged, the largest black hole having been a bigger source of gravitons and superparticles thereby permitting a greater curvature of spacetime.

The comparatively long duration of effects during the trial with the largest black hole that can be considered to have been constructed with the graphite was due to the collective string vibrations by all the molecules including the graphite atoms. Thus, the tube became exposed to a combined amount of radiated superparticles and gravitons, observed with the graphite tube having gained sufficient mass-energy to curve spacetime for the laser light to bend around it (Tahan 2011). The collective gravitational area was rare because the graphite tubes inconsistently leaked graphite to the acid. Without the additional graphite atoms, the compact area of energy did not appear; instead more commonly smaller black holes materialized that radiated less energy, smaller and less distinct laser light D-branes having been more frequent.

\section{Discussion}

Distinguishing the high gravitational areas in the tube as black holes was based on the redshifting visualization that was considered would be distinctly caused by a black hole--i.e. redshifting and the commencement of rotation seemingly due to the laser light entering the tube and interacting only with a specific region, consequently beginning frame dragging (Tahan 2011). The locations produced effects as would most high gravitational objects in the Cosmos, for instance altering a path of light due to the bending of spacetime (Tahan 2011), but were in small areas of the tube supporting that the objects most accurately should be described as black holes: massive, compact regions. Black hole and high gravitational area have been used interchangeably in this manuscript.

Creating D-brane appearance should not be thought to have been the focus of experimentation with the laser light, which was to form a conclusion regarding the pushing and pulling influences. The Figure 1 set-up should be remembered was for reaching a string vibration maximum due to a resonant frequency. A gradual increase in string vibrations due to the frequency was equivalent to a steady rise in energy. The appearance of the Figure 1 
D-brane was a surprise. By that, though the pushing influence was known and concluded likely to involve the graviphoton, the influence on spacetime thereby allowing for the observation of the D-brane with the open string was unimagined and particularly due to the largeness of the structure led to the understanding of spacetime to be a distinct medium to which properties can be transferred (Tahan 2015). Conclusions regarding branes were unanticipated when envisioning the possibility of an experiment.

The Figure 1 set-up was conceived by wondering what would happen if an atom aligned in a magnetic field was imparted quanta from an adjacent frequency source that would permit maintenance of the alignment. The thought involved imagining the atom gradually to fall apart due to the continuous quanta; the energy instead of simply passing through or being released would disrupt the structure of the body, which was appreciated to be an unconventional idea. Hydrogen was envisioned as an element for study due to the simplicity of the structure, which was thought could allow for various studies including concerning the forces of Nature if it under the influence of the quanta would be affected in a manner to suggest further investigation would be warranted.

Studies with Hydrogen are historical, but the literature seemed lacking in information on influencing Hydrogen with extremely low frequencies from adjacent sources--essentially no distance from the Hydrogen. Minimizing the distance between Hydrogen and the quanta was thought would be useful since a short antenna would not be a highly efficient, low frequency quanta source. By minimizing the distance, direct interaction of quanta with Hydrogen was imagined, reducing loss of quanta to the environment. The quanta could be understood to have a direct or more effective influence on the Hydrogen structure. The graphite tube replaced use of a Pyrex tube (No. 9825 ) in which a copper wire had been inserted in the acid as the antenna quanta supply. The conductive graphite tube served as an antenna and eased set-up. The conductive source $\mathrm{H}_{2} \mathrm{SO}_{4}$ of the Hydrogen was used since it was a conveniently available proton donor when including a frequency supply as the function generator, supplying the Hydrogen due to the $2 \mathrm{~Hz}$ current: $\approx 2.8 \mathrm{~mA}$ (maximum) with use of the graphite tube and $\approx 0.22 \mathrm{~A}$ (maximum) when using the Pyrex tube that involved $\mathrm{Vp}-\mathrm{p} \approx 12-12.5 \mathrm{~V}$ and $1-2 \mathrm{~mL} \mathrm{H}_{2} \mathrm{SO}_{4}$ per trial. Also, due to the $2 \mathrm{~Hz}$ with a specific amplitude the acid was an additional antenna. Different strong acids apparently could be substituted, pulling and pushing influences having been observed with use of hydrochloric acid in the Pyrex tube that was the holding vessel in trials with the loosely hanging pipette.

Mention of resonant frequency was for the string and should not have been confused with the resonance frequency of Hydrogen in relation to a magnetic field strength as used for nuclear magnetic resonance (NMR). The resonance frequency of elements for NMR may be a synergistic result of underlying, altered string vibrations. The change in tension of the brane in the magnetic field can be imagined as a slight altering of the brane shape, thereby changing the tension of the strings on the brane, so that the resonant frequency consequently was for the strings on the brane or for the brane generally. The change in tension should not be thought to have altered the vibration of a string enough to change the body that is due to the string vibration to a different particle.

The magnet was concluded eventually to alter string tension but originally was only to collect Hydrogen at a constant position, which initially was considered would reduce variables involving different distances from the frequency source. Later the positioning was thought useful for consistent graviton and superparticle emergence, especially for applications including directing the bodies to locations. The Hydrogen positioning also was considered could be helpful for isotope productions, being a consistent source of neutrons. The approximately 2000Gs magnet was initially available; being productive, continued use facilitated experimentation. Care was determined needed in selecting the magnetic field strength since excessive or insufficient change in brane tension was concluded would not allow for superstring symmetry breaking that should be comprehended as the primary success of the experiments without which events--black holes, spacetime bending, etc--would not have been observed. Change in tension--to limits--was thought to be linearly related to magnetic field strength, which could depend on the initial tension of the body under investigation. Again the string experimentation method was ineffective without the magnet.

Black holes have been proposed would appear when adding sufficient energy to an area ('t Hooft 2009). Similarly, the string vibrations due to the string experimentation method, an addition of energy to the area D-brane, led to a symmetry breaking allowing for gravitons to emerge in the Standard Model visible sector and interact with surroundings thereby producing the black holes, which had been unanticipated particularly to be influential enough to bend spacetime and alter a path of light (Tahan 2011). The pushing and pulling influences had occurred consistently to allow for the conclusion of an average rate of about $360 \mathrm{~s}$. By that, the set-up permitted periodic symmetry breakings in typical one hour trials. However, black hole visualizations depended on surroundings, e.g. extra graphite in the tube, including the potential for multiple symmetry breakings concurrently and therefore were inconsistent. Slighter effects due to less influential black holes could be missed. 
The largest D-brane with an open string and associated black hole of Figure 1 occurred at an unexpected time, though pushing and pulling influences had been recorded occasionally near the same time. So, the average rate for superstring symmetry breaking may need refinement.

\section{Conclusions}

String experimentation permits practical use of string resonance. The symmetry breaking can be thought as a restoring of supersymmetry to the visible sector. The release of superparticles and gravitons by the evaporating high gravitational areas, most precisely string black holes, can be considered a reconciling of quantum mechanics and gravity (Aharony, Gubser, Maldacena, Ooguri, \& Oz 2000). Apart from having explained that the size of the largest D-brane with an open string was due to evaporation of, radiated energy from, the biggest black hole in trials thereby demonstrating spacetime to be a specific medium and being supportive of AdS/CFT correspondence (Tahan 2015), the string black holes resolve the information paradox. Additionally, the black holes suggested that singularities need not exist.

Laboratory observations related to the emergence of gravitons and superparticles, especially the influences being fleeting, supported that the string experimentation method leads to a global symmetry breaking that allows the appearance of local symmetry, e.g. curving of spacetime by the tube in trials. Specifically, the ability to access the fifth dimension through Hydrogen can be understood to have been possible because of an underlying universal symmetry based on the string--apparent in the proton but understood throughout Nature if accepting the ubiquitous and consistent nature of spacetime (Tahan 2015), a unified field from which other fields emerge. Gravitons, more accurately supergravity, should not be considered apart from spacetime and vice versa.

When considering with only $2 \mathrm{~Hz}$ and a small amplitude a sufficiently high energy for deconfinement could be achieved by sourcing the energy through the proton with the vibrations of its strings, use of string vibrations to resolve global energy issues can be imagined. The ease and low cost of the set-up permits portable technological and industrial use apart from being a tool for research: for example to manufacture gravitons and superpartners as the gravitino (Tahan 2013).

\section{References}

Aharony, O., Gubser, S. S., Maldacena, J., Ooguri, H., \& Oz, Y. (2000). Large N field theories, string theory and gravity. Phys. Rept., 323, 183-386. http://dx.doi.org/10.1016/S0370-1573(99)00083-6

Anderson, P. W. (1963). Plasmons, gauge invariance, and mass. Phys. Rev., 130, 439-442. http://dx.doi.org/10.1103/PhysRev.130.439

ATLAS Collaboration (2012). Observation of a new particle in the search for the standard model Higgs boson with the ATLAS detector at the LHC. Phys. Lett., 716B, 1-29. http://dx.doi.org/10.1016/j.physletb.2012.08.020

Bergshoeff, E., Sezgin, E., \& Townsend, P. K. (1987). Supermembranes and eleven-dimensional supergravity. Phys. Lett., 189B, 75-78. http://dx.doi.org/10.1016/0370-2693(87)91272-X

Beringer, J. et al. (Particle Data Group) (2012). Review of particle physics. Phys. Rev., 86D, 010001. http://dx.doi.org/10.1103/PhysRevD.86.010001

Billó, M. et al. (2000). Shadow multiplets in AdS4/CFT3 and the super-Higgs mechanism. Nucl. Phys., 591B, 139-194. http://dx.doi.org/10.1016/S0550-3213(00)00532-0

Campanario, F., \& Kubocz, M. (2013). Higgs-boson production in association with three jets via gluon fusion at the LHC: Gluonic contributions. Phys. Rev., 88D, 054021. http://dx.doi.org/10.1103/PhysRevD.88.054021

CMS Collaboration (2012). Observation of a new boson at a mass of $125 \mathrm{GeV}$ with the CMS experiment at the LHC. Phys. Lett., 716B, 30-61. http://dx.doi.org/10.1016/j.physletb.2012.08.021

CMS Collaboration (2013). Observation of a new boson with mass near $125 \mathrm{GeV}$ in pp collisions at sqrt(s) $=7$ and 8 TeV. J. High Energy Phys., 06, 081. http://dx.doi.org/10.1007/JHEP06(2013)081

CMS Collaboration (2014). Evidence for the direct decay of the $125 \mathrm{GeV}$ Higgs boson to fermions. Nat. Phys., 10, 557-560. http://dx.doi.org/10.1038/nphys3005

Duff, M. J. \& Stelle, K. S. (1991). Multi-membrane solutions of D = 11 supergravity. Phys. Lett., 253B, 113-118. http://dx.doi.org/10.1016/0370-2693(91)91371-2

Englert, F. \& Brout, R. (1964). Broken symmetry and the mass of gauge vector mesons. Phys. Rev. Lett., 13, 321-323. http://dx.doi.org/10.1103/PhysRevLett.13.321 
Fradkin, E., \& Shenker, S. H. (1979). Phase diagrams of lattice gauge theories with Higgs fields. Phys. Rev., 19D, 3682-3697. http://dx.doi.org/10.1103/PhysRevD.19.3682

Georgi, H., \& Glashow, S. L. (1974). Unity of all elementary-particle forces. Phys. Rev. Lett., 32, 438-441. http://dx.doi.org/10.1103/PhysRevLett.32.438

Gross, D. J. \& Wilczek, F. (1973). Asymptotically free gauge theories. I. Phys. Rev., 8D, 3633-3652. http://dx.doi.org/10.1103/PhysRevD.8.3633

Guralnik, G. S., Hagen, C. R., \& Kibble, T. W. B. (1964). Global conservation laws and massless particles. Phys. Rev. Lett., 13, 585-587. http://dx.doi.org/10.1103/PhysRevLett.13.585

Higgs, P. W. (1964). Broken symmetries and the masses of gauge bosons. Phys. Rev. Lett., 13, 508-509. http://dx.doi.org/10.1103/PhysRevLett.13.508

Higgs, P. W. (1964). Broken symmetries, massless particles and gauge fields. Phys. Lett., 12(2), 132-133. http://dx.doi.org/10.1016/0031-9163(64)91136-9

Higgs, P. W. (1966). Spontaneous symmetry breakdown without massless bosons. Phys. Rev., 145, 1156-1163, http://dx.doi.org/10.1103/PhysRev.145.1156

Hořava, P., \& Witten, E. (1996). Eleven-dimensional supergravity on a manifold with boundary. Nucl. Phys., 475B, 94-114. http://dx.doi.org/10.1016/0550-3213(96)00308-2

Hořava, P., \& Witten, E. (1996). Heterotic and Type I string dynamics from eleven dimensions. Nucl. Phys., 460B, 506-524. http://dx.doi.org/10.1016/0550-3213(95)00621-4

Huq, M., \& Namazie, M.A. (1985). Kaluza-Klein supergravity in ten dimensions. Class. Quant. Grav,, 2, 293-308. http://dx.doi.org/10.1088/0264-9381/2/3/007

Krasnikov, N. V. (1987). On supersymmetry breaking in superstring theories. Phys. Lett., 193B, 37-40. http://dx.doi.org/10.1016/0370-2693(87)90452-7

LHC Higgs Cross Section Working Group (2013). Handbook of LHC Higgs Cross Sections: 3. Higgs Properties. arXiv: $1307.1347 \mathrm{v} 2$ [hep-ph].

Lunin, O., \& Mathur, S. D. (2002). AdS/CFT duality and the black hole information paradox. Nucl. Phys., 623B, 342-394, http://dx.doi.org/10.1016/S0550-3213(01)00620-4

Maldacena, J. (1998). The large $\mathrm{N}$ limit of superconformal field theories and supergravity. Adv. Theor. Math. Phys., 2, 231-252.

Martin, S. P. (2011). A supersymmetry primer. arXiv: hep-ph/9709356v6.

Meissner, K. A., Nilles, H. P., \& Olechowski, M. (1999). Supersymmetry breakdown at distant branes: The super-Higgs mechanism. Nucl. Phys., 561B, 30-42. http://dx.doi.org/10.1016/S0550-3213(99)00552-0

Mills, R. L. (1994). Space, time, and quanta: An introduction to contemporary physics, Part III. New York: W. H. Freeman.

Morita, Y., Nakano, H., \& Shimomura, T. (2012). Neutrino mass and proton decay in a U(1)R symmetric model. arXiv: $1212.4304 \mathrm{v} 1$ [hep-ph].

Scherk, J., \& Schwarz, J. H. (1974). Dual models and the geometry of space-time. Phys. Lett., 52B, 347-350, http://dx.doi.org/10.1016/0370-2693(74)90059-8

Scherk, J., \& Schwarz, J. H. (1974). Dual models for non-hadrons. Nucl. Phys., 81B, 118-144. http://dx.doi.org/10.1016/0550-3213(74)90010-8

Scherk, J., \& Schwarz, J. H. (1975). Dual field theory of quarks and gluons. Phys. Lett., 57B, 463-466. http://dx.doi.org/10.1016/0370-2693(75)90269-5

Schwarz, J. H. (1980). Algebraic structure of broken supersymmetry. Nucl. Phys., 173B, 311-318. http://dx.doi.org/10.1016/0550-3213(80)90221-7

Schwarz, J. H. (1982). Superstring theory. Phys. Rept., 89, 223-322. http://dx.doi.org/10.1016/0370-1573(82)90087-4

Schwinger, J. (1962). Gauge invariance and mass. Phys. Rev., 125, 397-398. http://dx.doi.org/10.1103/PhysRev.125.397

't Hooft, G. (1974). A planar diagram theory for strong interactions. Nucl. Phys., 72B, 461-473. 
http://dx.doi.org/10.1016/0550-3213(74)90154-0

't Hooft, G. (2009). Dimensional reduction in quantum gravity. arXiv: gr-qc/9310026v2.

Tahan, A. C. (2011). Exposing strings in the laboratory with a novel technique. Appl. Phys. Res., 3(2), 39-51. http://dx.doi.org/10.5539/apr.v3n2p39

Tahan, A. C. (2012). Diagrammatic presentation for the production of gravitons and supersymmetry. Mod. Appl. Sci., 6(9), 76-83. http://dx.doi.org/10.5539/mas.v6n9p76

Tahan, A. C. (2013). Low mass gravitino: Re-introducing the superpartner as dark matter with consideration to inflation due to experimentation. Mod. Appl. Sci., 7(12), 43-55. http://dx.doi.org/10.5539/mas.v7n12p43

Tahan, A. C. (2014). BICEP2 and the gravitino mass: The questionable result. Mod. Appl. Sci., 8(5), 30-35. http://dx.doi.org/10.5539/mas.v8n5p30

Tahan, A. C. (2015). Spacetime: A distinct medium. Earth Sci. Res., 4(1), 40-46. http://dx.doi.org/10.5539/esr.v4n1p40

Takayama, F., \& Yamaguchi, M. (2000). Gravitino dark matter without r-parity. Phys. Lett. 485B, 388-392. http://dx.doi.org/10.1016/S0370-2693(00)00726-7

Volkov, D. V., \& Soroka, V. A. (1973). Higgs effect for Goldstone particles with spin 1/2. JETP Lett., 18, 312-314.

Weinberg, S. (1973). Non-Abelian gauge theories of the strong interactions. Phys. Rev. Lett., 31, 494-497. http://dx.doi.org/10.1103/PhysRevLett.31.494

Yang, C. N., \& Mills, R. L. (1954). Conservation of isotopic spin and isotopic gauge invariance. Phys. Rev., 96, 191-195. http://dx.doi.org/10.1103/PhysRev.96.191

Zwiebach, B. (2004). A first course in string theory. Cambridge: Cambridge University Press.

\section{Copyrights}

Copyright for this article is retained by the author(s), with first publication rights granted to the journal.

This is an open-access article distributed under the terms and conditions of the Creative Commons Attribution license (http://creativecommons.org/licenses/by/3.0/). 